\title{
Repurposing FDA-Approved Phytomedicines, Natural Products, Antivirals and Cell Protectives against SARS-CoV-2 (COVID-19) RNA-Dependent RNA Polymerase
}

\author{
Mahmoud Kandeel ${ }^{\text {Corresp., } 1}$, Yukio Kitade ${ }^{2}$, Abdullah Almubarak ${ }^{3}$ \\ ${ }^{1}$ King Faisal University, Al Ahsa, Al Ahsa, Saudi Arabia \\ 2 Gifu University, gifu, Japan \\ ${ }^{3}$ King Faisal University, Al Ahsa,31982, Al Ahsa, Saudi Arabia \\ Corresponding Author: Mahmoud Kandeel \\ Email address: mahmoud.kandeal@vet.kfs.edu.eg
}

Following the recent emergence of SARS-CoV-2 or coronavirus disease 2019 (COVID-19), drug discovery and vaccine design to combat this fatal infection are critical. In this study, an essential enzyme in the SARS-CoV-2 replication machinery, RNA-dependent RNA polymerase (RDRP), is targeted in a virtual screening assay using a set of 1664 FDAapproved drugs, including sets of botanical and synthetic derivatives. A set of 22 drugs showed a high docking score of >-7. Notably, approximately one-third of the top hits were either from natural products or biological molecules. The FDA-approved phytochemicals were sennosides, digoxin, asiaticoside, glycyrrhizin, neohesperidin, taxifolin, quercetin and aloin. These approved natural products and phytochemicals are used as general tonics, antioxidants, cell protectives, and immune stimulants (nadid, thymopentin, asiaticoside, glycyrrhizin) and in other miscellaneous systemic or topical applications. A comprehensive analysis was conducted on standard precision and extra precision docking, two-step molecular dynamics simulations, binding energy calculations and a post dynamics analysis. The results reveal that two drugs, docetaxel and neohesperidin, showed strong binding profiles with SARS CoV-2 RdRP. These results can be used as a primer for further drug discovery studies in the treatment of COVID-19. This initiative repurposes safe FDAapproved drugs against COVID-19 RdRP, providing a rapid channel for the discovery and application of new anti-CoV therapeutics. 
1 Repurposing FDA-Approved Phytomedicines, Natural

2

3

4

\section{0}

\title{
Products, Antivirals and Cell Protectives against SARS-CoV-2 (COVID-19) RNA-Dependent RNA Polymerase
}

\author{
Mahmoud Kandeel ${ }^{1,2, *}$, Yukio Kitade ${ }^{3,4}$, Abdullah I. Al-Mubarak ${ }^{5}$ \\ ${ }^{1}$ Department of Biomedical Sciences, College of Veterinary Medicine, King Faisal University, \\ Al-Hofuf, 31982 Al-Ahsa, Saudi Arabia; \\ ${ }^{2}$ Department of Pharmacology, Faculty of Veterinary Medicine, Kafrelshikh University, \\ Kafrelshikh 33516, Egypt \\ ${ }^{3}$ Department of Applied Chemistry, Faculty of Engineering, Aichi Institute of Technology, 1247 \\ Yachigusa, Yakusa-cho, Toyota, Aichi 470-0392, Japan \\ ${ }^{4}$ Department of Chemistry and Biomolecular Sciences, Faculty of Engineering, Gifu University, \\ 1-1 Yanagido, Gifu 501-1193, Japan \\ ${ }^{5}$ Department of Microbiology, College of Veterinary Medicine, King Faisal University, Alhofuf, \\ Alahsa 31982, Saudi Arabia
}

\section{Corresponding Author:}

Mahmoud Kandeel

Email: mkandeel@kfu.edu.sa 


\section{Abstract}

26 Following the recent emergence of SARS-CoV-2 or coronavirus disease 2019 (COVID-19), drug 27 discovery and vaccine design to combat this fatal infection are critical. In this study, an essential enzyme in the SARS-CoV-2 replication machinery, RNA-dependent RNA polymerase (RDRP), is targeted in a virtual screening assay using a set of 1664 FDA-approved drugs, including sets of botanical and synthetic derivatives. A set of 22 drugs showed a high docking score of >-7. Notably, approximately one-third of the top hits were either from natural products or biological molecules. The FDA-approved phytochemicals were sennosides, digoxin, asiaticoside, glycyrrhizin, neohesperidin, taxifolin, quercetin and aloin. These approved natural products and phytochemicals are used as general tonics, antioxidants, cell protectives, and immune stimulants (nadid, thymopentin, asiaticoside, glycyrrhizin) and in other miscellaneous systemic or topical applications. A comprehensive analysis was conducted on standard precision and extra precision docking, two-step molecular dynamics simulations, binding energy calculations and a post dynamics analysis. The results reveal that two drugs, docetaxel and neohesperidin, showed strong binding profiles with SARS CoV-2 RdRP. These results can be used as a primer for further drug discovery studies in the treatment of COVID-19. This initiative repurposes safe FDA-approved drugs against COVID-19 RdRP, providing a rapid channel for the discovery and application of new anti-CoV therapeutics.

\section{Key words}

SARS-CoV-2; COVID-19; polymerase; virtual screening

\section{Abbreviations}

FDA, Food and Drug Administration; Severe Acute Respiratory Syndrome Coronavirus-2, SARS-CoV-2; coronavirus disease 2019, COVID-19; RNA-dependent RNA polymerase, RdRP; Middle East Respiratory Syndrome Coronavirus, MERS CoV; spike, S; envelope, E; membrane, M; nucleocapsid, N; Global initiative on sharing all influenza data, GISAID.

\section{INTRODUCTION}


53 Coronavirus disease 2019 (COVID-19) appeared in the Hubei prefecture of China in December 542019 and spread widely within a month, with cases recorded in more than 2 countries (WHO 2020). The causative agent was determined to be a new coronavirus, SARS-CoV-2.

56 SARS-CoV-2 is a member of the Betacoronavirus genus (Liu et al.), a group of RNA viruses 57 characterized by the presence of a polyprotein and a relatively large genome ( $\sim 30 \mathrm{~kb})$ that codes 58 for multiple structural and nonstructural proteins. The four structural proteins include spike (S), 59 envelope (E), membrane (M) and nucleocapsid (N) (Shi et al. 2015). Approximately 14-16 nonstructural proteins are encoded at the 5'-terminal of the polyprotein. The proteins are essential for virus replication, mRNA synthesis, and processing of viral proteins (Brian \& Baric 2005). Coronaviruses have recently evolved from a causative agent of mild respiratory symptoms to severely pathogenic forms. Three major pathogenic and fatal CoVs have emerged in the last two decades: SARS CoV, MERS CoV, and lastly, SARS-CoV-2 (Guarner 2020).

RNA-dependent RNA polymerase (RdRP) is a nonstructural protein that is crucial for SARSCoV-2 replication (Gaurav \& Al-Nema 2019). RdRP is a significant drug target against CoVs, implemented for the discovery of new antivirals against MERS CoV (Gordon et al. 2020), dengue (Shimizu et al. 2019), Zika (Ahmad et al. 2020), influenza A (Niazi et al. 2019) and hepatitis C viruses (Dash et al. 2019).

In this study, SARS-CoV-2 RdRP was used to virtually screen a dataset of safe, non-toxic and FDA-approved pharmacological compounds. The drugs with a high score can be repurposed to treat SARS-CoV-2, taking advantage of its safety and direct applicability without further toxicity or preclinical testing. In addition, the phylogenetic relationships between SARS-CoV-2 RdRP and those of the viruses responsible for other recent coronavirus epidemics, SARS and MERS $\mathrm{CoV}$, were investigated. Full molecular and dynamics profiles of drugs binding to RdRP were evaluated by combining docking and Molecular Dynamics (MD) simulations.

\section{MATERIALS AND METHODS}

\section{Construction of FDA approved drugs dataset}

A total of 1664 FDA approved drugs were retrieved from commercial compounds databases at 
82 using OPLS2005 force field at physiological $\mathrm{pH}$. The compounds dataset are provided in the 83 Supplementary files 1.

\section{SARS-CoV-2 RdRP optimization and structure preparation}

85 The protein preparation module in Maestro software package (Schrodinger LLC, NY, USA) was used to optimize the protein structure for docking. The protein (PDB ID 7BV2) was protonated, optimized at cellular $\mathrm{pH}$ conditions, and energy minimized using OPLS2005 force field. All ions, except the active site magnesium, and non-relevant crystallographic materials were removed. During preparation, two structural files were prepared comprising RdRP in the presence or absence of magnesium ions. The docking grid was generated by the selection of active site residues. The docking box was centered around the active site residues ASP255 and ASP256 with a size of $20 \AA$. For comparative purpose, the sequence similarity between SARS CoV, SARS CoV-2 and MERS CoV RdRPs were retrieved and aligned (supplementary methods file). The reliability of docking procedures were assigned by redocking of the co- crystalized inhibitor (remdesivir). The obtained poses were comparable to the co-crystalized structure (Fig. 1).

\section{Virtual screening}

The glide docking module of Schrodinger maestro package was used for virtual screening. Twostep docking protocol was carried out. At first, the docking was set to run under the standard precision (SP docking) (Friesner et al., 2004; Halgren et al., 2004). The results were ranked by the docking score. Remdesivir was used as standard RdRP inhibitor. It is a broad-spectrum antiviral with potent inhibition of MERS CoV RdRP (Gordon et al. 2020). Compounds with a docking score of -7.00 or higher were retrieved and reanalyzed by extra-precision docking protocol (XP-docking). Magnesium ions are important in coordinating the polymerase actions of RdRP. Therefore, the two docking interactions were run comprising the presence and absence of $\mathrm{Mg}$ ions in their binding sites.

\section{Molecular dynamics simulations}

Groningen Machine for Chemical Simulations (GROMACS) 5.1.4. software was used in all molecular dynamics (MD) simulations (Abraham et al. 2015; Van Der Spoel et al. 2005). The ligand parameter, topology and restraint and the protein were handled by AMBERFF14SB force field and general AMBER force field (GAFF), respectively. The RdRP-ligand complexes were 
111 solvated in a cubic box with $1.0 \mathrm{~nm}$ from protein to box edge. The box was filled with a single 112 point charge (SPC) water model. The solvated complexes were minimized for 5000 steps.

113 During water and ions coupling, the heavy atoms of protein and ligand were restrained. Two-step 114 system equilibration was adopted comprising 50ps NVT at 300K followed by 1ns NPT ensemble 115 at $300 \mathrm{~K}$. Production stages were done over simulation times of 20 and 100 ns with NPT 116 ensemble. Parrinello-Rahman algorithm maintain constant pressure at 1 bar and V-rescale 117 thermostat algorithmfor temperature coupling at 300K. Long-range electrostatics under periodic 118 conditions with a direct space cut-off $12 \AA$ were restrained by Particle Mesh Ewald (PME) 119 method. Time step was set to 2 fs. Coordinates and output values were collected every 10 ps.

\section{Trajectory analysis}

121 GROMACS MD simulation toolkits were used in the trajectory analysis. The g_rms and g_rmsf 122 were used to calculate the root mean square deviation (RMSD) of the starting structure to the end 123 of the simulation time and the per-residue root mean square fluctuation (RMSF) of the protein 124 residues.

\section{Binding energy calculations}

126 The molecular mechanics-generalized Born surface area (MM/GBSA) was calculated by the 127 g_mmpbsa tool.

\section{Statistical analysis}

129

130

131

132

133

134

135

136

Multiple correlation comparisons were performed between the obtained docking score and the properties and interactions of the compounds with SARS-CoV-2, including the molecular weight, the hydrogen bonding and the lipophilic interaction parameters. The analysis was carried out with the Pearson's correlation coefficient, implemented in GraphPad Prism version 7 (GraphPad Software, La Jolla, CA, USA).

\section{RESULTS}

Virtual screening and Docking 
137 The virtual screening data for 1664 FDA-approved drugs against SARS-CoV-2 are provided in

138 Supplementary Table 1. The presented parameters include the docking scores, ligand 139 efficiencies, and lipophilic and hydrogen bonding interactions. The assessment of docking 140 protocol was confirmed by docking of remdesivir monophosphate (RMP). The redocked pose 141 was compared with the co-crystalized ligand (Fig. 1). It is was evident that the presence of 142 magnesium ions increased the docking scores of RMP in both of the standard precision and 143 extra-precision docking. In the absence of magnesium ions, the SP and XP docking scores of 144 RMP were -8.9 and -9.7 , respectively. These scores was decreased by about $15 \%$ in the absence 145 of magnesium ions. In contrast, the docked ligands from the FDA approved drugs dataset 146 showed higher scores in the absence of magnesium ions. This might be attributed to the 147 favourable interaction of magnesium ions with the phosphorylated substrates.

148 Table 1 shows the 26 compounds with the highest docking scores, which were -7 or higher. 149 Within the top hits, there were five general tonics, cell protectives, and antioxidants; one 150 antiviral; one anti-parasitic; one antibiotic; one immune stimulant; three anticancer drugs; and 151 other miscellaneous systemically or topically acting drugs.

152 A considerable number of natural products, natural product derivatives, and biological molecules 153 were present among the top hits. Plant flavonoids (quercetin, troxerutin, taxifolin), plant 154 glycosides (sennosides A and B, digoxin), a citrus-derived sweetener (neohesperidin), and 155 biological molecules (oxiglutatione, nadid) constituted about $36 \%$ of the top binding drugs with 156 SARS-CoV-2 RdRP.

157 In order to get deeper insights into the interaction of drugs with SARS-CoV-2 RdRP, the binding 158 mode and ligand interaction profiles of the drugs and also of remdesivir were examined (Fig. 2).

159 The active site residues comprised positively charged amino acids (LYS476, ARG507, LYS681 160 and GLU694,), negatively charged amino acids (ASP328, ASP501, ASP506, ASP643, ASP644 161 and ASP748), aromatic residues (TRP461, TYR474, TRP500, TYR502, TRP683 and PHE726), 162 polar amino acids (GLN327, ASN330, SER475, SER565, SER642, CYS696, SER697, GLN698 163 and PRO715) and non-polar amino acids (GLY329, ALA313, ALA331, ILE333, VAL471, 164 ILE472, GLY473, MET484, LEU641 and ALA645). Naringen formed two hydrogen bonds with 165 ARG555 and THR591 (Fig. 2A). In contrast, echinacoside showed multiple interaction routes, 166 including six hydrogen bonds with ARG555, CYC622, ASP623, SER682, ASP760 and ARG836 
167 (Fig. 2B). It was noticeable that the number of hydrogen bonds formed between the docked 168 ligands with the active is affected by the presence of magnesium ions. While metal coordinates is 169 evident with the docked poses, this was underscored by lower hydrogen bonds interactions (Fig. 170 2). This might contributed to the observed lower docking scores in structures containing 171 magnesium.

172 Statistical analysis revealed a significant positive correlation between the docking score and the 173 hydrogen bond, lipophilic interaction, and ligand efficiency scores $(p<.001$; Table 2$)$. There was 174 a negative low but significant correlation between the obtained docking scores and the molecular 175 weights of the compounds. This explains the superior docking scores of the selected set of drugs 176 over the standard inhibitor remdesivir, which involved the lower molecular weights and 177 improved hydrogen bonding and lipophilic interactions.

178 For further filtration to identify the most potent hits, compounds with a score of -7.00 or higher 179 were subjected to XP-docking followed by MM/GBSA calculations (Table 3). Six compounds 180 produced high docking scores exceeding -11.00: echinacoside, salvianolic acid B, ginsenoside, 181 neohesperidin, troxerutin, and docetaxel. These top six were then used for further MD 182 simulations.

\section{Molecular dynamics simulations for 20 ns}

184 Combining docking with MD simulation is a powerful tool for drug discovery, as these 185 techniques rank compounds on their binding affinity and can also evaluate target-receptor binding energy and drug-receptor dynamics. In this study, the top-ranked compounds from XPdocking were subjected to MD simulation followed by post dynamic analysis of binding energy. A two-step filtering process was adopted. First, the six compounds were run in MD simulation for $20 \mathrm{~ns}$ and their RMSD, RMSF, and MM/GBSA values evaluated. In the second stage, the top two compounds were tested in a more comprehensive $100 \mathrm{~ns}$ MD simulation. Structural changes in the backbone residues of RdRP relative to the initial structure (RMSD) were compared after 20 ns simulations (Fig. 3).

According to the MM/GBSA binding energies, strong binding was indicated for docetaxel (195 $69.19 \mathrm{kcal} / \mathrm{mol})$ and neohesperidin $(-65.754 \mathrm{kcal} / \mathrm{mol})$. Ginsenoside showed a moderate binding 
196

197

198

199

200

201

202

203

204

205

206

207

208

209

210

211

212

213

214

215

216

217

218

219

220

221

222

223

224

energy of $-47.52 \mathrm{kcal} / \mathrm{mol}$ (Table 4). The other three compounds yielded low binding energy values, all less than $-30.708 \mathrm{kcal} / \mathrm{mol}$. In addition, all of the tested compounds showed low structure RMSD during the $20 \mathrm{~ns}$ simulations, with values $<0.24 \mathrm{~nm}$ (Table 4). Based on the 20 ns simulation data, docetaxel and neohesperidin were selected for further analysis in $100 \mathrm{~ns}$ MD simulations.

\section{Molecular dynamics simulations for $100 \mathrm{~ns}$}

In order to obtain deeper insight into the strongest-binding drugs, the two with binding energy $>$ $60 \mathrm{kcal} / \mathrm{mol}$ (docetaxel and neohesperidin) were subjected to $100 \mathrm{~ns}$ MD simulations followed by analysis of binding free energy, RMSD, RMSF, hydrogen bond length, and radius of gyration. The estimated MM/GBSA binding energies were -67.273 and -63.669 for docetaxel and neohesperidin, respectively. These values imply superior binding for both of the compounds.

The $100 \mathrm{~ns}$ simulations yielded average RMSD values of 0.25 and $0.22 \mathrm{~nm}$ for docetaxel and neohesperidin, respectively. These values indicate stability of RdRP in complexes with docetaxel or neohesperidin. Both RMSD and per-residue RMSF showed almost similar profiles for RdRP complexes with docetaxel and neohesperidin (Fig. 4).

\section{Radius of gyration}

Radius of gyration can be taken as a measure of the compactness of the system. Fig. 5 shows Rg variations observed during $100 \mathrm{~ns}$ MD simulations. For both compounds, the average Rg value was $3.18 \mathrm{~nm}$. High Rg values indicate lower compactness or more unfolded protein, while low $\mathrm{Rg}$ indicates more stable structures. The similar Rg values indicate comparable stability of the two examined drugs when complexed with RdRP.

\section{Decomposition of MM/GBSA binding energy at $100 \mathrm{~ns}$}

Post-dynamic energy decomposition analysis was used to evaluate the dominant interactions during recognition of the drugs by RdRP (Table 5). The results revealed that van der Waals and electrostatic interactions were the predominant forces underlying binding of the drugs to RdRP. Specifically, van der Waals interactions were the major forces for both docetaxel and neohesperidin, followed by electrostatic interactions. 


\section{The binding site of docetaxel and neohesperidin}

226 The binding characteristics of docetaxel and neohesperidin with RdRP are provided in Fig. 6.

227 The binding site is mostly composed of hydrophobic residues (ILE548, VAL557, ILE589,

228 THR680, and ALA688), with the presence of few positively charged residues (LYS551,

229 LYS545, and ARG555), negatively charged residues (ASP623 and ASP761), and neutral

230 residues (SER549, SER682, ASN691, SER759, and SER814). The interaction of docetaxel and

231 neohesperidin with RdRP was respectively supported by six and seven hydrogen bonds.

232 Neohesperidin formed hydrogen bonding with ASP618, SER682, SER759, while docetaxel 233 interacted with ILE548, SER549, LYS551 and SER814.

\section{The number of hydrogen bonds}

235 The number of hydrogen bonds between docetaxel and neohesperidin and RdRP was traced 236 during MD simulation (Fig. 7 and supplementary Fig. 3). The average number of hydrogen 237 bonds were 4.3 and 6.2 for docetaxel and neohesperidin, respectively. Additionally, the distance 238 between the two drugs and two selected residues in the active site was traced during simulation 239 (Fig. 7B and supplementary Fig. 4). The selected residues were LYS521 for docetaxel and 240 ASP588 for neohesperidin. After initial stabilization within the first few ns, the results indicated 241 the lack of large fluctuations in the position of both drugs in the active site.

\section{DISCUSSION}

This work covers a knowledge gap about the SARS-CoV-2 RdRP and its relation to the two previous fatal CoVs, SARS-CoV and MERS-CoV. Additionally, the structure of RdRP was targeted by a set of FDA-approved drugs for the purpose of relocating already approved and safe drugs for SARS-CoV-2 treatment.

Based on their pharmacokinetic aspects, some of these top hits are not appropriate for systemic application due to their low levels of absorption e.g., sennosides (Leng-Peschlow 1986) and pyrantel pamoate (Ryan 2018). Others have specific applications with an impact on specific body systems or disorders, such as digoxin (cardiovascular system), acarbose (antidiabetic), venetoclax and taxifolin (anticancer drugs), and chlorhexidine (a drug for topical application). 
253 Ritonavir is the only antiviral predicted to bind with SARS-CoV-2 RdRP. It is approved for HIV 254 treatment and acts by inhibition of the virus protease.(Croxtall \& Perry 2010) Ritonavir was in 255 the $6^{\text {th }}$ rank of the top hits. A recent clinical report showed the improvement of an index case 256 with pneumonia with a significant decrease in the virus load after ritonavir/lopinavir 257 treatment.(Lim et al. 2020) The drugs with higher docking scores, such as nadid or thymopentin, 258 are general body and immune stimulants that might be of value in COVID-19 treatment.

259 Remdesivir was used as a reference drug for binding with RdRP. It successfully inhibited 260 MERS-CoV RdRP. The docking output of remdesivir had a score of -5.98 , which was lower 261 than the scores of the 26 drugs selected that had a high affinity for RdRP.

262 Docetaxel complex with SARS-CoV-2 RdRP showed high stability and free binding energy. 263 This interaction is driven by favourable van der Waals and electrostatic interactions, and includes 264 the formation of about six hydrogen bonds with RdRP active site residues. By MD simulation for $265100 \mathrm{~ns}$, docetaxel formed an average of 4.3 hydrogen bonds. Docetaxel has large mw (807.9 $266 \mathrm{~g} / \mathrm{mol}$ ). However, this drug obeys the Lipiniski's rule of five in LogP and favourable hydrogen 267 donor count ( 5 or lower). In addition, docetaxel is a cytotoxic agent with clinical approval in the 268 treatment of different types of cancers, e.g. breast and prostate cancers (Lyseng-Williamson \& 269 Fenton 2005; Tannock et al. 2004).

270 Neohesperidin is a flavanone glycoside from citrus fruits with wide applicability in vascular 271 diseases and cancer treatment trials (Walker et al. 2014). Similar to docetaxel, a favourable 272 hydrogen bonding profile and electrostatic and van der Waals interactions support the potent 273 binding of neohesperidin with SARS CoV-2 RdRP. By MD simulation for $100 \mathrm{~ns}$, neohesperidin 274 formed an average of 4.3 hydrogen bonds.

275 Taking the docking and MD simulation results together, the two compounds exhibited 276 favourable binding features comprising low RMSD, high binding free energy, low radius of 277 gyration, formation of several hydrogen bonds, strong electrostatic interactions, and the presence 278 of polar and hydrophobic components in the interaction with RdRP. Based on these findings, 279 docetaxel and neohesperidin are most likely to be strong inhibitors of SARS CoV-2 RdRP. 
282 After the molecular modeling and virtual screening of approximately 1664 FDA-approved drugs, 283 a set that interact strongly with SARS CoV-2 RdRP has been formed and is provided. These are 284 suggested for use as an addition to the standard treatment. The superiority of obtained values 285 over a known MERS-CoV inhibitor further supports the merit of repurposing these compounds 286 against SARS CoV-2 RdRP. Furthermore, the binding potency and molecular dynamics of docetaxel and neohesperidin in particular recommends them for such use. However, further assessment by antiviral assays is required.

\section{ACKNOWLEDGMENTS}

291

The authors extend their appreciation to the Deputyship for Research \& Innovation, Ministry of 292 Education in Saudi Arabia for funding this research work through the project number IFT20110.

\section{ADDITIONAL INFORMATION AND DECLARATIONS}

295

296 297 298

299 300

301 302 303 304 305 306

\section{Funding}

This project is funded by the Deputyship for Research \& Innovation, Ministry of Education in Saudi Arabia for funding this research work through the project number IFT20110.

\section{Competing Interests}

The authors declare that they have no competing interests.

\section{Author contributions}

Mahmoud Kandeel conceived and designed the experiments, performed the experiments, analyzed the data, prepared figures and/or tables, authored or reviewed drafts of the paper, and approved the final draft.

Yukio Kitade conceived and designed the experiments, performed the experiments, analyzed the data, prepared figures and/or tables, authored or reviewed drafts of the paper, and approved the final draft. 
307

308

309

310

311

312

313

314

315

316

317

318

319

320

321

322

323

324

325

326

327

328

329

330

331

332

333

Abdullah I. Al-Mubarak conceived and designed the experiments, performed the experiments, analyzed the data, prepared figures and/or tables, authored or reviewed drafts of the paper, and approved the final draft.

\section{Data availability}

All data are within the manuscript and the supplementary materials.

3

\section{References}

Abraham MJ, Murtola T, Schulz R, Páll S, Smith JC, Hess B, and Lindahl E. 2015. GROMACS: High performance molecular simulations through multi-level parallelism from laptops to supercomputers. SoftwareX 1:19-25.

Ahmad N, Rehman AU, Badshah SL, Ullah A, Mohammad A, and Khan K. 2020. Molecular dynamics simulation of zika virus NS5 RNA dependent RNA polymerase with selected novel nonnucleoside inhibitors. Journal of Molecular Structure 1203:127428.

Brian D, and Baric R. 2005. Coronavirus genome structure and replication. Coronavirus replication and reverse genetics: Springer, 1-30.

Croxtall JD, and Perry CM. 2010. Lopinavir/Ritonavir: a review of its use in the management of HIV-1 infection. Drugs 70:1885-1915. 10.2165/11204950-000000000-00000

Dash S, Aydin Y, and Stephens CM. 2019. Hepatitis C Virus NS5B RNA-Dependent RNA Polymerase Inhibitor: An Integral Part of HCV Antiviral Therapy. Viral Polymerases: Elsevier, 211-235.

Gaurav A, and Al-Nema M. 2019. Polymerases of Coronaviruses: Structure, Function, and Inhibitors. Viral Polymerases: Elsevier, 271-300.

Gordon CJ, Tchesnokov EP, Feng JY, Porter DP, and Gotte M. 2020. The antiviral compound remdesivir potently inhibits RNA-dependent RNA polymerase from Middle East respiratory syndrome coronavirus. Journal of Biological Chemistry:jbc. AC120. 013056.

Guarner J. 2020. Three Emerging Coronaviruses in Two DecadesThe Story of SARS, MERS, and Now COVID-19. American Journal of Clinical Pathology. 
334 335

336

337

338

339

340

341

342

343

344

345

346

347

348

349

350

351

352

353

354

355

356

357

358

359

360

361

362

363

364

365

Leng-Peschlow E. 1986. Dual effect of orally administered sennosides on large intestine transit and fluid absorption in the rat. Journal of Pharmacy and Pharmacology 38:606-610. 10.1111/j.20427158.1986.tb03089.x

Lim J, Jeon S, Shin H-Y, Kim MJ, Seong YM, Lee WJ, Choe K-W, Kang YM, Lee B, and Park S-J. 2020. Case of the Index Patient Who Caused Tertiary Transmission of Coronavirus Disease 2019 in Korea: the Application of Lopinavir/Ritonavir for the Treatment of COVID-19 Pneumonia Monitored by Quantitative RT-PCR. Journal of Korean Medical Science 35.

Liu Z, Xiao X, Wei X, Li J, Yang J, Tan H, Zhu J, Zhang Q, Wu J, and Liu L. Composition and divergence of coronavirus spike proteins and host ACE2 receptors predict potential intermediate hosts of SARS-CoV-2. Journal of Medical Virology.

Lyseng-Williamson KA, and Fenton C. 2005. Docetaxel. Drugs 65:2513-2531.

Niazi K, Rabizadeh S, Le Ny A-L, Buzko O, Golovato J, and Soon-Shiong P. 2019. Small molecule inhibitors of influenza A RNA-dependent RNA polymerase. Google Patents.

Ryan ET. 2018. 296 - Antiparasitic Agents. In: Long SS, Prober CG, and Fischer M, eds. Principles and Practice of Pediatric Infectious Diseases (Fifth Edition): Elsevier, 1567-1587.e1562.

Shi J, Zhang J, Li S, Sun J, Teng Y, Wu M, Li J, Li Y, Hu N, and Wang H. 2015. Epitope-Based Vaccine Target Screening against Highly Pathogenic MERS-CoV: An In Silico Approach Applied to Emerging Infectious Diseases. PloS one 10:e0144475.

Shimizu H, Saito A, Mikuni J, Nakayama EE, Koyama H, Honma T, Shirouzu M, Sekine S-i, and Shioda T. 2019. Discovery of a small molecule inhibitor targeting dengue virus NS5 RNA-dependent RNA polymerase. PLoS Neglected Tropical Diseases 13.

Tannock IF, De Wit R, Berry WR, Horti J, Pluzanska A, Chi KN, Oudard S, Théodore C, James ND, and Turesson I. 2004. Docetaxel plus prednisone or mitoxantrone plus prednisone for advanced prostate cancer. New England Journal of Medicine 351:1502-1512.

Van Der Spoel D, Lindahl E, Hess B, Groenhof G, Mark AE, and Berendsen HJ. 2005. GROMACS: fast, flexible, and free. Journal of computational chemistry 26:1701-1718.

Walker R, Janda E, and Mollace V. 2014. Chapter 84 - The Use of Bergamot-Derived Polyphenol Fraction in Cardiometabolic Risk Prevention and its Possible Mechanisms of Action. In: Watson RR, Preedy VR, and Zibadi S, eds. Polyphenols in Human Health and Disease. San Diego: Academic Press, $1087-1105$.

WHO. 2020. https://www.who.int/emergencies/diseases/novel-coronavirus-2019. 
Figure legends

Fig. 1. Docking of remdesivir with COVID-19 CoV RdRP. A) The docking site of remdesivir monophosphate into the active site of RdRP. B) The docking site showing the superimposed conformations of the co-crystalized ligand and the docked pose. The docked pose is coloured by red and the co-crystalized ligand is coloured by the atom type. C) The ligand interactions of remdesivir monophosphate with the active site of RdRP.

Fig. 2. The docking mode and ligand interactions of naringen, echinacoside and salvianolic acid with SARS-CoV-2 RdRP, in the presence or absence of magnesium ions. A) Ligand interactions of naringen with RdRP in the absence of magnesium ions B) Ligand interactions of naringen with RdRP in the presence of magnesium ions C) Ligand interactions of echinacoside with RdRP in the absence of magnesium ions D) Ligand interactions of echinacoside with RdRP in the presence of magnesium ions E) Ligand interactions of salvianolic acid with RdRP in the absence of magnesium ions F) Ligand interactions of salvianolic acid with RdRP in the presence of magnesium ions. Charged residue (negative) in pink, positive charged residue in blue, hydrophobic residues in cyan, hydrogen bonds: purple arrow, stacking interactions: green sticks, metal coordinates: grey sticks.

Fig. 3. Molecular dynamics simulation for $20 \mathrm{~ns}$. The RMSD during $20 \mathrm{~ns}$ simulation of the top 6 compounds after XP-docking. Apo implies SARS CoV-2 RdRP without any ligands.

Fig. 4. Molecular dynamics simulation for $100 \mathrm{~ns}$. The RMSD during $100 \mathrm{~ns}$ simulation of the top 2 compounds after MD simulation for $100 \mathrm{~ns}$, docetaxel and neohesperidin. A) RMSD of docetaxel and neohesperidin B) The per-residue RMSF of RdRP bound with docetaxel and neohesperidin. The figure includes the average of three different experiments.

Fig. 5. Radius of gyration of RdRP bound with docetaxel and neohesperidin after $100 \mathrm{~ns}$ molecular dynamics simulation. The figure includes the average of three different experiments. 
394 Fig. 6. The ligand interactions of docetaxel and neohesperidin with SARS CoV-2 RdRP 395 from the average structure after $100 \mathrm{~ns}$ molecular dynamics simulation. A) Docetaxel B) 396 Neohesperidin.

397 Fig. 7. Molecular dynamics simulation for 100 ns. The number of hydrogen bonds and 398 distance from RdRP residues during $100 \mathrm{~ns}$ simulation of the top 2 compounds after MD 399 simulation for $100 \mathrm{~ns}$, docetaxel and neohesperidin. A) The number of hydrogen bonds formed by docetaxel and neohesperidin B) The distance between the two drugs and two 401 selected residues in the active site during $100 \mathrm{~ns}$ MD simulation. The selected residues were LYS521 for docetaxel and ASP588 for neohesperidin.

403

404

405

406

407

408

409

410

411

412

413

414

415

416

417

Supplementary Fig. 1. Molecular dynamics simulation for $100 \mathrm{~ns}$. The RMSD during 100 ns simulation of the top 2 compounds after MD simulation for $20 \mathrm{~ns}$, docetaxel and neohesperidin. A) RMSD of docetaxel and neohesperidin B) The per-residue RMSF of RdRP bound with docetaxel and neohesperidin. The average from the three experiments is displayed in Fig. 4.

Supplementary Fig. 2. Radius of gyration of RdRP bound with docetaxel and neohesperidin after $100 \mathrm{~ns}$ molecular dynamics simulation. The average from the three experiments is displayed in Fig. 5.

Supplementary Fig. 3. The number of hydrogen bonds formed between docetaxel and neohesperidin during molecular dynamics simulation for 100 ns. A) The number of hydrogen bonds formed by docetaxel B) The number of hydrogen bonds formed by neohesperidin. The average from the three experiments is displayed in Fig. 7.

Supplementary Fig. 4. The distance between the two drugs and two selected residues in the active site during $100 \mathrm{~ns}$ MD simulation. The selected residues were LYS521 for docetaxel and ASP588 for neohesperidin. 


\section{Table 1 (on next page)}

Table 1: Natural compounds showing the highest docking score.

Table 1: Natural compounds showing the highest docking score (>-7) after docking of a set of FDA approved drugs against SARS-CoV-2 RNA-dependent RNA polymerase 
Table 1: Natural compounds showing the highest docking score (>-7) after docking of a set of FDA approved drugs against

2 SARS-CoV-2 RNA-dependent RNA polymerase

\begin{tabular}{|c|c|c|c|c|c|c|c|c|}
\hline Name & $\begin{array}{c}\text { Mol. } \\
\text { Weight }\end{array}$ & $\begin{array}{c}\text { docking } \\
\text { score }\end{array}$ & $\begin{array}{l}\text { glide ligand } \\
\text { efficiency }\end{array}$ & $\begin{array}{l}\text { glide } \\
\text { lipo }\end{array}$ & $\begin{array}{l}\text { glide } \\
\text { hbond }\end{array}$ & $\begin{array}{l}\text { glide } \\
\text { evdw }\end{array}$ & source & Clinical uses \\
\hline Sennoside B & 862.7 & -8.1 & -0.1 & -0.6 & -0.2 & -58.8 & $\begin{array}{l}\text { Glycoside from } \\
\text { Senna plants }\end{array}$ & Constipation \\
\hline Digoxin & 780.9 & -7.8 & -0.1 & -2.4 & -0.2 & -62.4 & $\begin{array}{c}\text { Glycoside from } \\
\text { digitalis }\end{array}$ & cardiovascular \\
\hline Asiaticoside & 959.1 & -7.6 & -0.1 & -1.8 & 0.0 & -50.4 & $\begin{array}{c}\text { triterpenoid fro } \\
\text { m Centella } \\
\text { asiatica }\end{array}$ & antioxidant \\
\hline $\begin{array}{c}\text { Glycyrrhizin } \\
\text { (Glycyrrhizic Acid) }\end{array}$ & 822.9 & -7.6 & -0.1 & -0.8 & 0.0 & -55.6 & $\begin{array}{l}\text { triterpene glycos } \\
\text { ide from } \\
\text { liquorice }\end{array}$ & $\begin{array}{l}\text { Hepatoprotecti } \\
\text { ve, food } \\
\text { sweetener }\end{array}$ \\
\hline $\begin{array}{c}\text { Neohesperidin } \\
\text { dihydrochalcone } \\
(\text { Nhdc })\end{array}$ & 612.6 & -7.5 & -0.2 & -1.4 & -0.1 & -46.7 & $\begin{array}{c}\text { flavanone glyco } \\
\text { side in citrus } \\
\text { fruits }\end{array}$ & food sweetener \\
\hline $\begin{array}{c}\text { Taxifolin } \\
\text { (Dihydroquercetin) }\end{array}$ & 304.3 & -7.4 & -0.3 & -1.3 & -0.4 & -23.7 & $\begin{array}{c}\text { Flavonoid } \\
\text { present in many } \\
\text { plants }\end{array}$ & anticancer \\
\hline $\begin{array}{c}\text { Quercetin } \\
\text { (Sophoretin) }\end{array}$ & 302.2 & -7.1 & -0.3 & -1.1 & -0.4 & -25.1 & $\begin{array}{c}\text { Flavonoid in } \\
\text { citrus fruits }\end{array}$ & $\begin{array}{l}\text { PI3K, PKC, } \\
\text { Src, Sirtuin }\end{array}$ \\
\hline Aloin (Barbaloin) & 418.4 & -7.0 & -0.2 & -1.5 & -0.3 & -28.3 & $\begin{array}{c}\text { Glycoside from } \\
\text { Aloe }\end{array}$ & Tyrosinase \\
\hline
\end{tabular}

3

4 Synthetic drugs showing the highest docking score (>-7) after docking of a set of FDA approved drugs against SARS-CoV-2 5 RNA-dependent RNA polymerase 


\begin{tabular}{|c|c|c|c|c|c|c|c|}
\hline Name & $\begin{array}{c}\text { Mol. } \\
\text { Weight }\end{array}$ & $\begin{array}{c}\text { docking } \\
\text { score }\end{array}$ & $\begin{array}{c}\text { glide } \\
\text { ligand } \\
\text { efficiency }\end{array}$ & $\begin{array}{c}\text { glide } \\
\text { lipo }\end{array}$ & $\begin{array}{c}\text { glide } \\
\text { hbond }\end{array}$ & $\begin{array}{c}\text { glide } \\
\text { evdw }\end{array}$ & Clinical uses \\
\hline Nadid(NAD+) & 663.4 & -8.2 & -0.2 & -0.9 & -0.5 & -56.2 & Chronic fatigue/general tonic \\
\hline Leucovorin & 601.6 & -8.1 & -0.2 & -0.3 & -1.5 & -32.2 & folate analog \\
\hline Thymopentin & 679.8 & -7.9 & -0.2 & -1.6 & -1.5 & -50.3 & $\begin{array}{c}\text { Immune stimulant } \\
\text { \&Inflammation related }\end{array}$ \\
\hline Ritonavir & 720.9 & -7.7 & -0.2 & -2.9 & -0.3 & -67.9 & HIV Protease \\
\hline Venetoclax & 868.4 & -7.5 & -0.1 & -2.5 & -0.6 & -64.0 & Bcl-2 inhibitor \\
\hline Oxiglutatione & 612.6 & -7.4 & -0.2 & -0.8 & -0.8 & -48.2 & Cell protection \\
\hline Iopamidol & 777.1 & -7.4 & -0.2 & -0.9 & -1.1 & -37.1 & Contrast agent \\
\hline Acarbose & 645.6 & -7.3 & -0.2 & -1.2 & -0.3 & -37.2 & Type II diabetes \\
\hline Chlorhexidine HCl & 578.4 & -7.3 & -0.2 & -1.9 & -0.4 & -39.7 & Topical antiseptic \\
\hline Salvianolic acid B & 718.6 & -7.3 & -0.1 & -1.2 & 0.0 & -55.3 & Others \\
\hline Troxerutin & 742.7 & -7.2 & -0.1 & -1.0 & -0.5 & -42.1 & Thrombin \\
\hline Cefodizime Sodium & 628.6 & -7.2 & -0.2 & -1.0 & -0.9 & -44.0 & antibiotic \\
\hline (-)Epicatechin & 290.3 & -7.1 & -0.3 & -1.0 & -0.6 & -22.0 & Others \\
\hline Ioversol & 807.1 & -7.1 & -0.2 & -0.7 & -1.1 & -38.1 & Contrast dyes \\
\hline Pyrantel Pamoate & 594.7 & -7.1 & -0.2 & -1.9 & -0.2 & -34.4 & antiparasitic \\
\hline Cobicistat & 776.0 & -7.0 & -0.1 & -2.8 & -0.4 & -63.7 & P450 (e.g. CYP17) \\
\hline
\end{tabular}




\section{Table 2 (on next page)}

Statistical correlation analysis of docking output.

Pearson`s correlation of the obtained docking score with the drugs mw and interation parameters with SARS-CoV-2 RdRP. 
1

2 Table 2. Pearson's correlation of the obtained docking score with the drugs mw and interation

3 parameters with SARS-CoV-2 RdRP

\begin{tabular}{|c|c|c|c|c|c|}
\hline & $\begin{array}{l}\text { docking score } \\
\text { vs. } \\
\text { MolWeight }\end{array}$ & $\begin{array}{l}\text { docking } \\
\text { score } \\
\text { vs. } \\
\text { glide ligand } \\
\text { efficiency }\end{array}$ & $\begin{array}{l}\text { docking score } \\
\text { vs. } \\
\text { glide lipo }\end{array}$ & $\begin{array}{l}\text { docking } \\
\text { score } \\
\text { vs. } \\
\text { glide hbond }\end{array}$ & $\begin{array}{l}\text { docking score } \\
\text { vs. } \\
\text { glide evdw }\end{array}$ \\
\hline \multicolumn{6}{|l|}{ Pearson $r$} \\
\hline $\mathrm{r}$ & -0.08911 & 0.3242 & 0.1453 & 0.3442 & 0.1221 \\
\hline $\begin{array}{l}95 \% \text { confidence } \\
\text { interval }\end{array}$ & -0.17 to -0.04 & 0.28 to 0.37 & 0.098 to 0.19 & 0.3 to 0.38 & 0.07 to 0.17 \\
\hline R squared & 0.008 & 0.11 & 0.021 & 0.12 & 0.015 \\
\hline \multicolumn{6}{|l|}{$P$ value } \\
\hline P (two-tailed) & 0.0003 & $<0.0001$ & $<0.0001$ & $<0.0001$ & $<0.0001$ \\
\hline$P$ value summary & **** & $* * * *$ & $* * * *$ & $* * * *$ & $* * * *$ \\
\hline $\begin{array}{l}\text { Significant? } \\
(\text { alpha }=0.05)\end{array}$ & Yes & Yes & Yes & Yes & Yes \\
\hline $\begin{array}{l}\text { Number of XY } \\
\text { Pairs }\end{array}$ & 1657 & 1657 & 1657 & 1657 & 1657 \\
\hline
\end{tabular}

4

5

6

7

8 


\section{Table 3(on next page)}

The docking scores and estimated binding energy after XP-docking protocol.

The compounds were raked by their docking scores. 
1 Table 3. The docking scores and estimated binding energy after XP-docking protocol

\begin{tabular}{|l|c|c|}
\hline Name & $\begin{array}{c}\text { docking score in the } \\
\text { absence of } \mathrm{Mg}+2\end{array}$ & $\begin{array}{c}\text { Docking scores in the } \\
\text { presence of } \mathrm{Mg}+2\end{array}$ \\
\hline Echinacoside & -14.544 & -12.648 \\
\hline Salvianolic acid B & -13.803 & -11.174 \\
\hline Ginsenoside Re & -12.685 & -10.258 \\
\hline Neohesperidin & -12.023 & -9.704 \\
\hline Troxerutin & -11.465 & -9.174 \\
\hline Docetaxel & -11.114 & -7.48 \\
\hline Diosmin & -10.99 & -8.115 \\
\hline Acarbose & -10.972 & -8.595 \\
\hline Rutin (Rutoside) & -10.883 & -8.813 \\
\hline Asiaticoside & -10.555 & -10.763 \\
\hline Naringin (Naringoside) & -10.555 & -6.732 \\
\hline Dihydrostreptomycin & -10.401 & -6.967 \\
\hline Hesperidin & -10.389 & -9.286 \\
\hline Neomycin sulfate & -10.387 & -8.159 \\
\hline Fluvastatin sodium & -9.826 & -9.173 \\
\hline Maltitol & -9.639 & -5.516 \\
\hline Lactobionic acid & -9.383 & -6.431 \\
\hline Amikacin disulfate & -9.327 & -7.947 \\
\hline Isepamicin Sulphate & -9.244 & -7.946 \\
\hline Oleuropein & -9.21 & -6.072 \\
\hline Pravastatin sodium & -8.557 & -8.438 \\
\hline & & \\
\hline & & \\
\hline
\end{tabular}


Table 4 (on next page)

Post dynamic analysis of RMSD.

Average RMSD and the MM-GBSA binding energy after 20 ns MD simulation for the top six compounds after XP-docking. 
1 Table 4. Average RMSD and the MM-GBSA binding energy after $20 \mathrm{~ns}$ MD simulation for 2 the top six compounds after XP-docking.

3

\begin{tabular}{|c|c|c|c|c|c|c|c|}
\hline & Apo & Docetaxel & $\begin{array}{c}\text { Echinacosi } \\
\text { de }\end{array}$ & $\begin{array}{c}\text { Ginsen } \\
\text { oside }\end{array}$ & $\begin{array}{c}\text { Neohesperi } \\
\text { din }\end{array}$ & $\begin{array}{c}\text { Salvianolic } \\
\text { acid B }\end{array}$ & Troxerutin \\
\hline $\begin{array}{c}\text { Average RMSD } \\
(\mathrm{nm})\end{array}$ & 0.194 & 0.220 & 0.241 & 0.234 & 0.176 & 0.235 & 0.236 \\
\hline $\begin{array}{c}\text { MM-GBSA } \\
(\mathrm{kcal} / \mathrm{mol})\end{array}$ & -69.19 & -30.708 & -47.52 & -65.754 & -14.015 & -30.116 \\
\hline
\end{tabular}

4 


\section{Table 5 (on next page)}

Energy decomposition analysis.

Decomposition of the estimated MMGBSA binding energy for the binding of docetaxel and neohesperidin with SARS CoV-2 RdRP. 
1 Table 5. Decomposition of the estimated MMGBSA binding energy for the binding of 2 docetaxel and neohespridin with SARS CoV-2 RdRP.

\begin{tabular}{|l|r|r|}
\hline & docetaxel & neohespridin \\
\hline van der Waal energy & -158.953 & -169.743 \\
\hline Electrostattic energy & -19.672 & -111.149 \\
\hline Polar solvation energy & 130.971 & 238.858 \\
\hline SASA energy & 19.581 & -21.655 \\
\hline Binding energy & -67.273 & -63.669 \\
\hline
\end{tabular}

3 


\section{Figure 1}

Docking results.

Docking of remdesivir with COVID-19 CoV RdRP. A) The docking site of remdesivir monophosphate into the active site of RdRP. B) The docking site showing the superimposed conformations of the co-crystalized ligand and the docked pose. The docked pose is coloured by red and the co-crystalized ligand is coloured by the atom type. C) The ligand interactions of remdesivir monophosphate with the active site of RdRP.
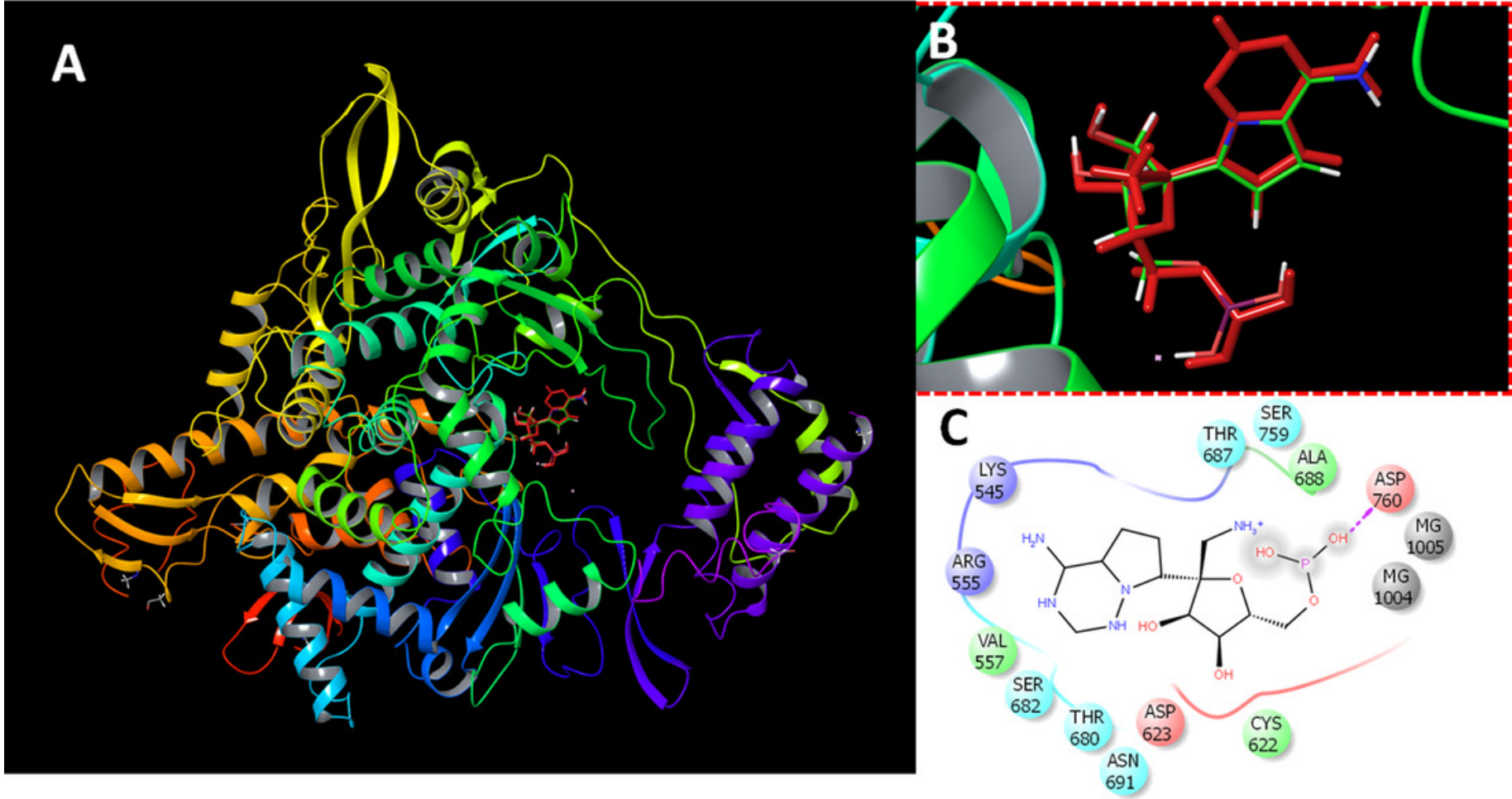


\section{Figure 2}

Docking results in the presence or absence of magnesium ions.

The docking mode and ligand interactions of naringen, echinacoside and salvianolic acid with SARS-CoV-2 RdRP, in the presence or absence of magnesium ions. A) Ligand interactions of naringen with RdRP in the absence of magnesium ions B) Ligand interactions of naringen with RdRP in the presence of magnesium ions $\mathrm{C}$ ) Ligand interactions of echinacoside with RdRP in the absence of magnesium ions D) Ligand interactions of echinacoside with RdRP in the presence of magnesium ions E) Ligand interactions of salvianolic acid with RdRP in the absence of magnesium ions F) Ligand interactions of salvianolic acid with RdRP in the presence of magnesium ions. Charged residue (negative) in pink, positive charged residue in blue, hydrophobic residues in cyan, hydrogen bonds: purple arrow, stacking interactions: green sticks, metal coordinates: grey sticks. 
A
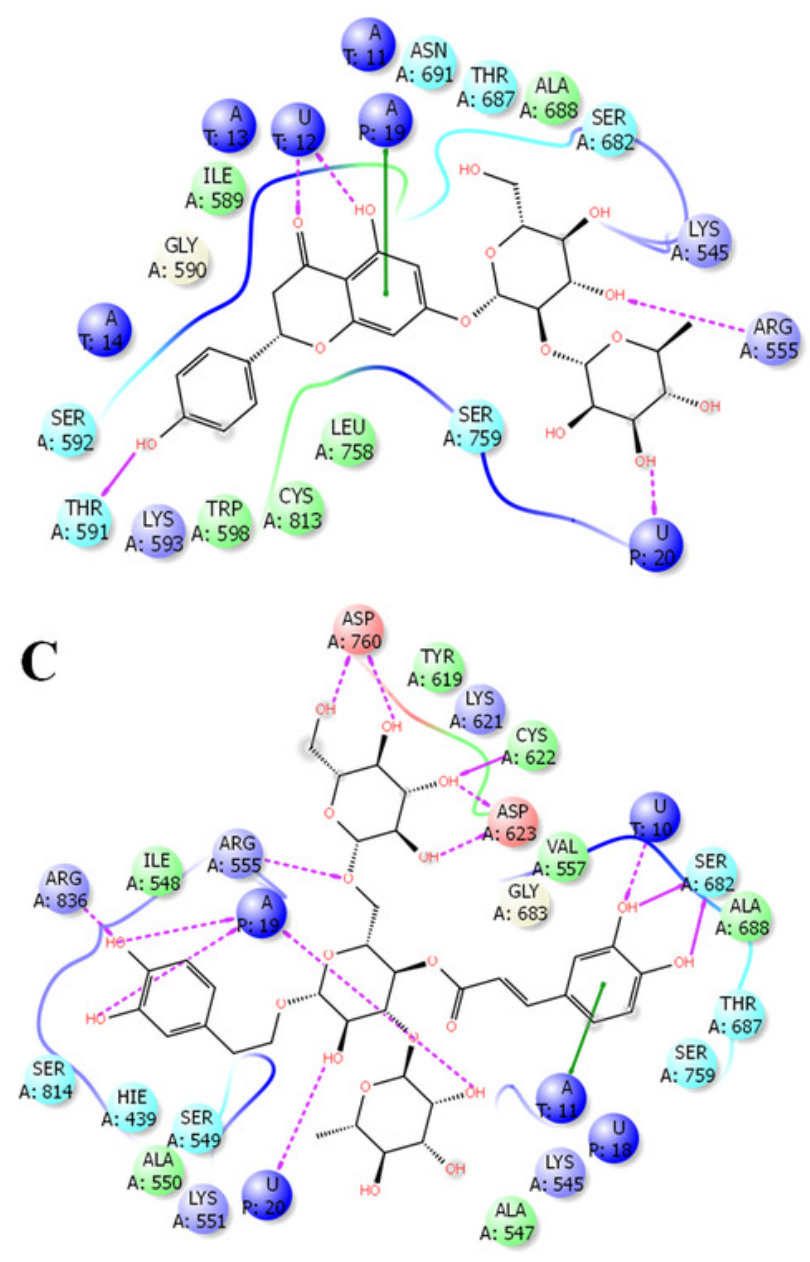

E

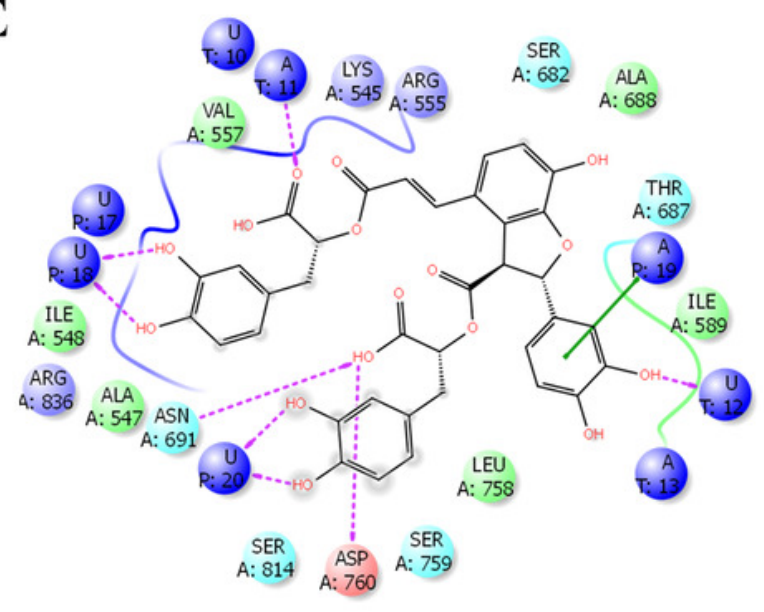

B

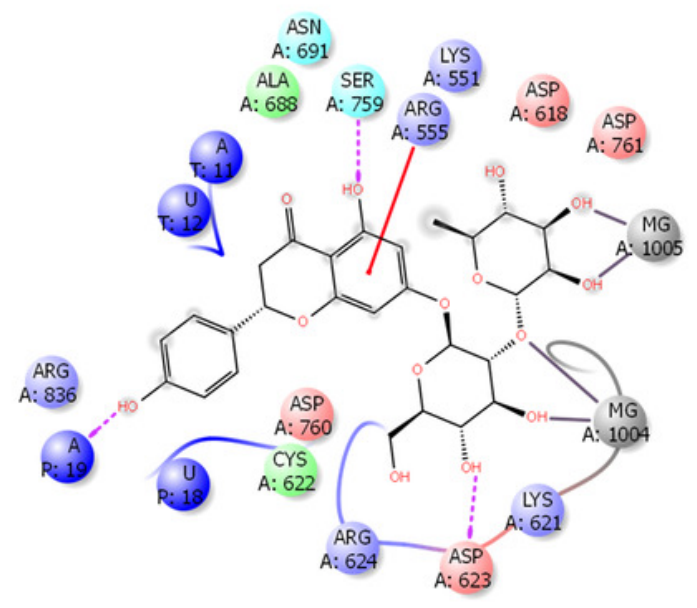

D
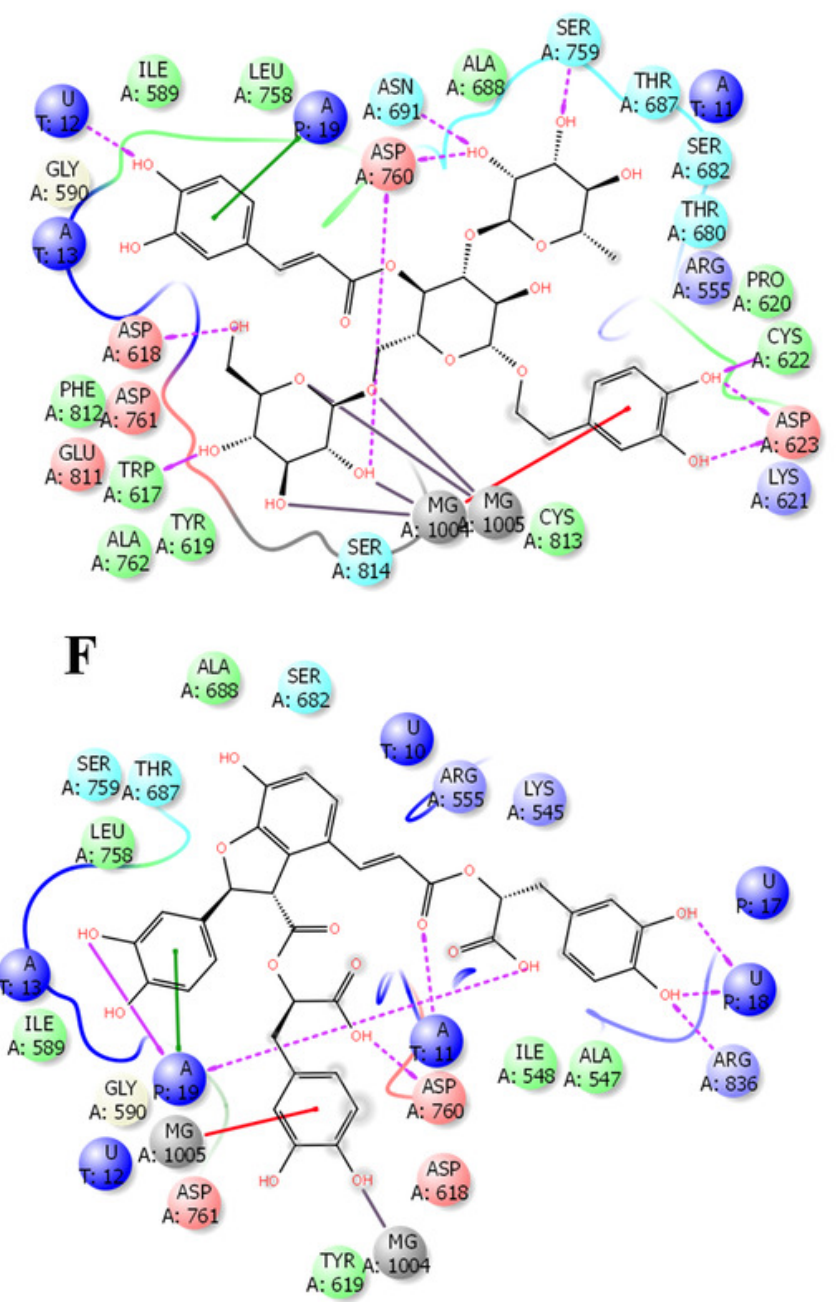
Figure 3

Molecular dynamics simulation for 20 ns.

The RMSD during 20 ns simulation of the top 6 compounds after XP-docking. Apo implies SARS CoV-2 RdRP without any ligands. 

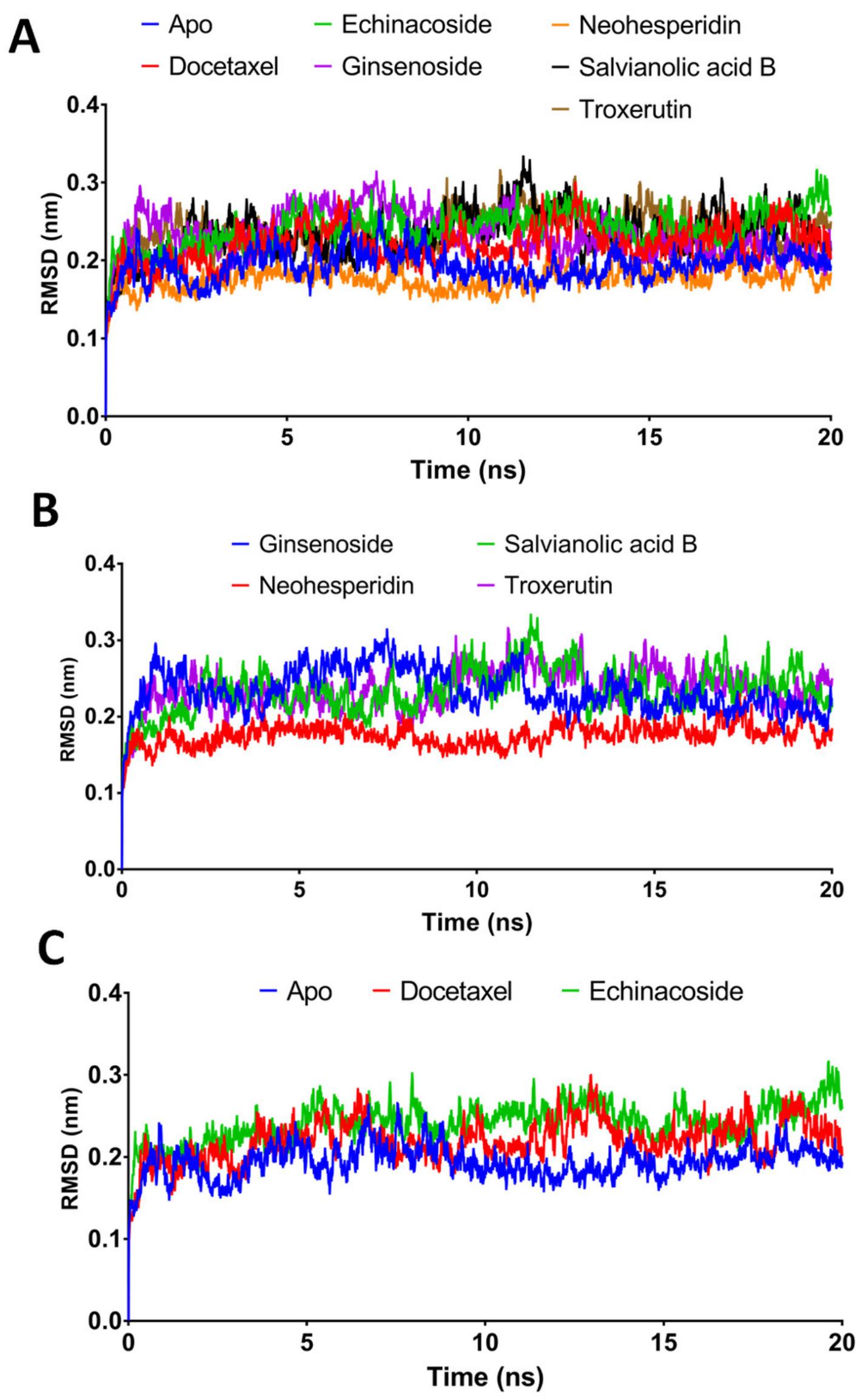
Figure 4

Molecular dynamics simulation for 100 ns.

The RMSD during 100 ns simulation of the top 2 compounds after MD simulation for 100 ns, docetaxel and neohesperidin. A) RMSD of docetaxel and neohesperidin B) The per-residue RMSF of RdRP bound with docetaxel and neohesperidin. The figure includes the average of three different experiments. 
A

- Docetaxel - Neohesperidin

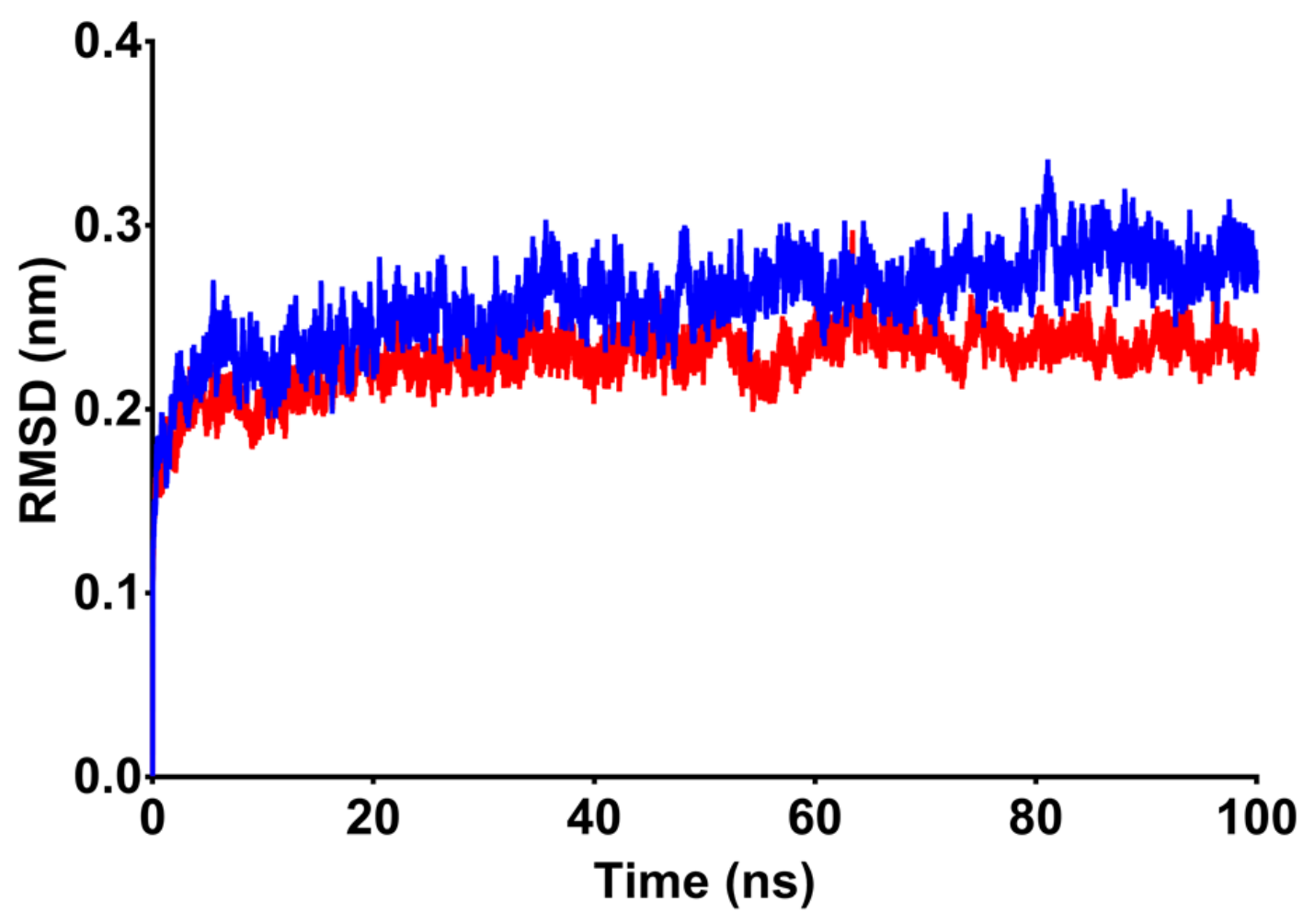

B

- Docetaxel - Neohesperidin

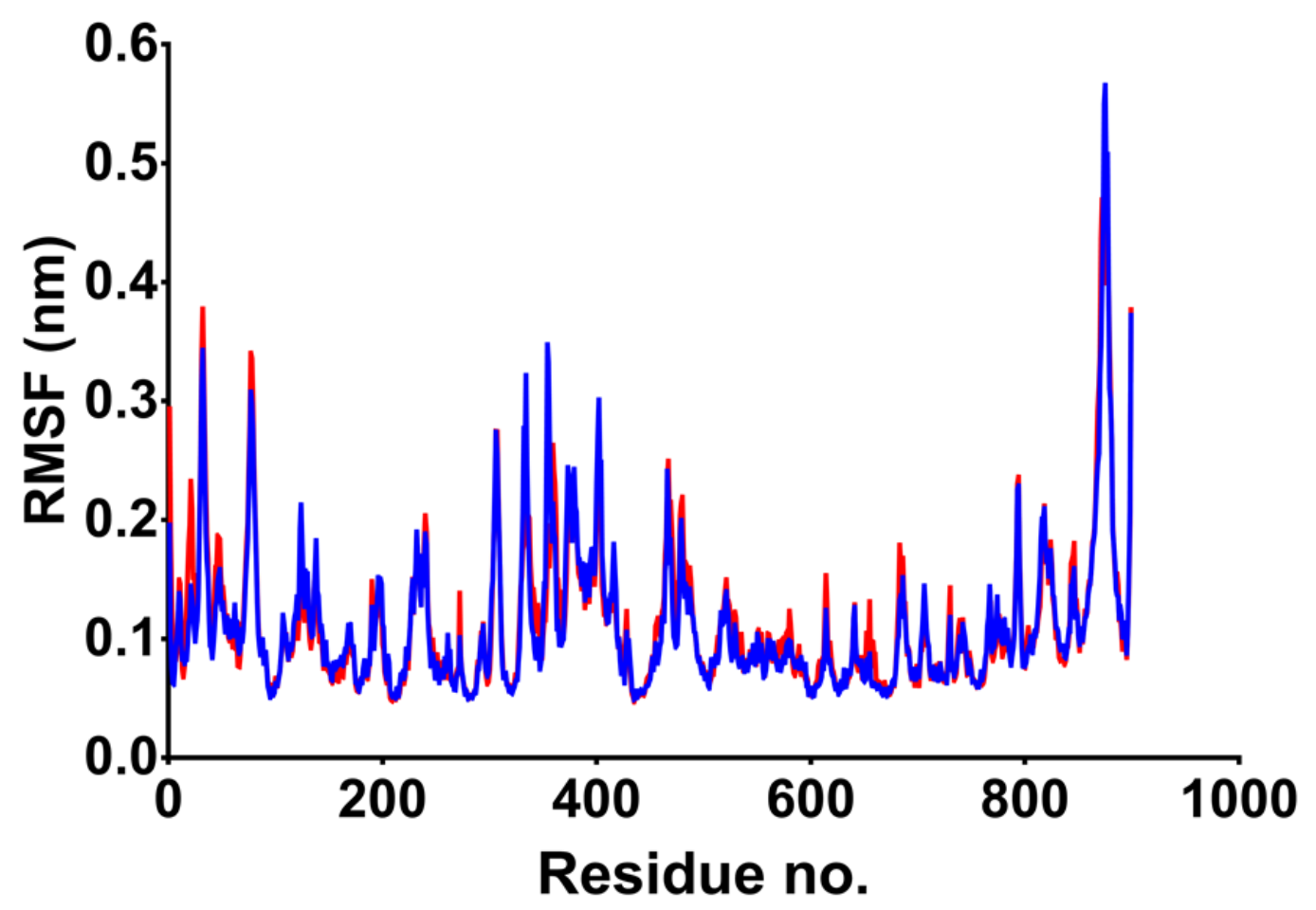




\section{Figure 5}

Molecular dynamics simulation for 100 ns.

Radius of gyration of RdRP bound with docetaxel and neohesperidin after 100 ns molecular dynamics simulation. The figure includes the average of three different experiments.
- Docetaxel1
- Neohesperidin1

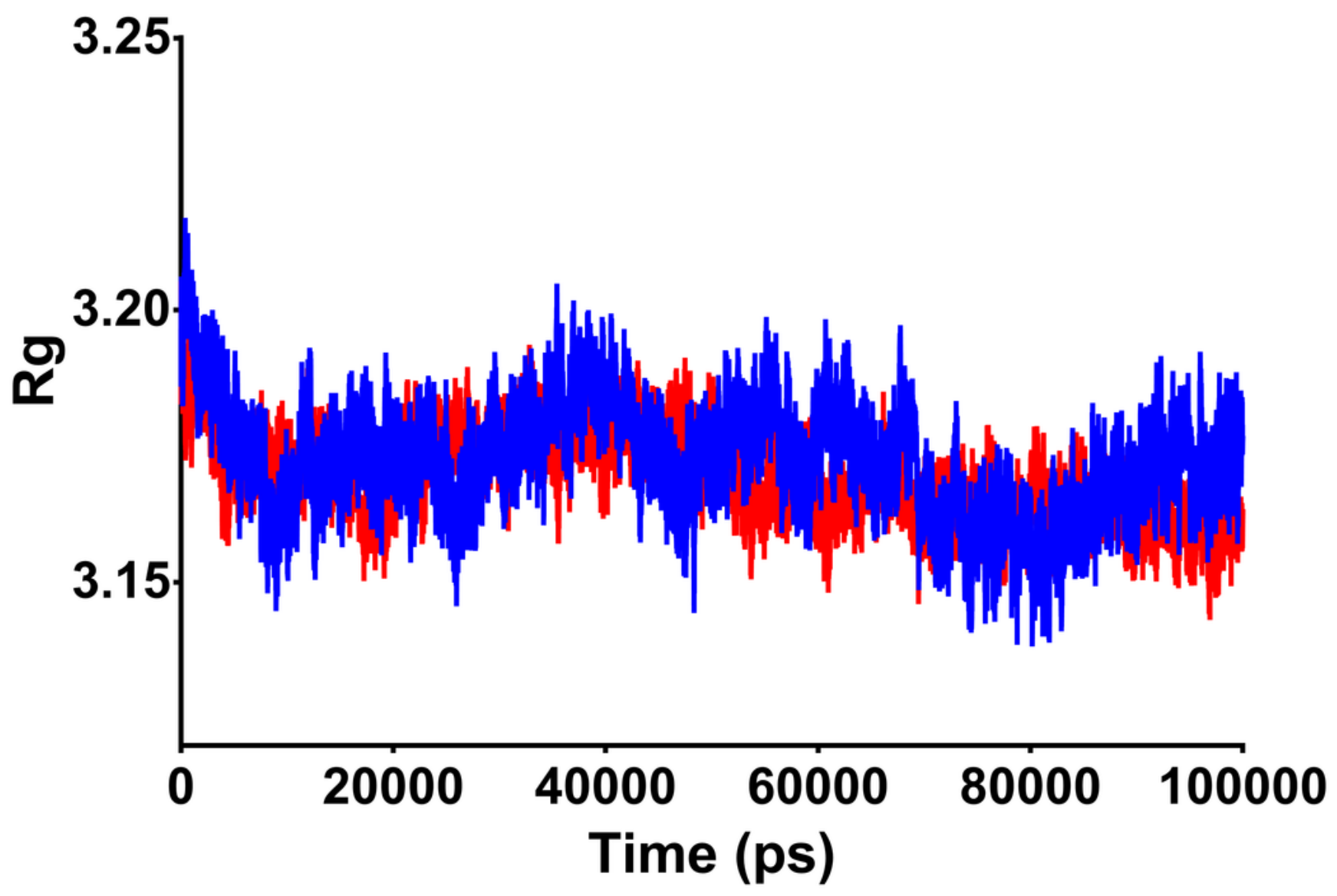


Figure 6

Molecular dynamics simulation for $100 \mathrm{~ns}$.

The ligand interactions of docetaxel and neohesperidin with SARS CoV-2 RdRP from the average structure after 100 ns molecular dynamics simulation. A) Docetaxel B) Neohesperidin.

A

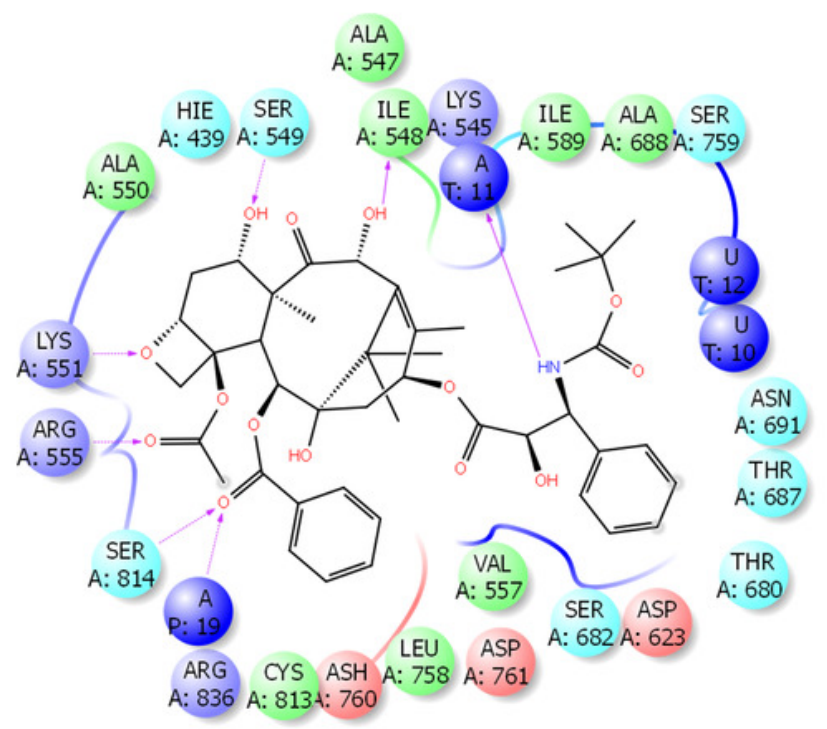

B

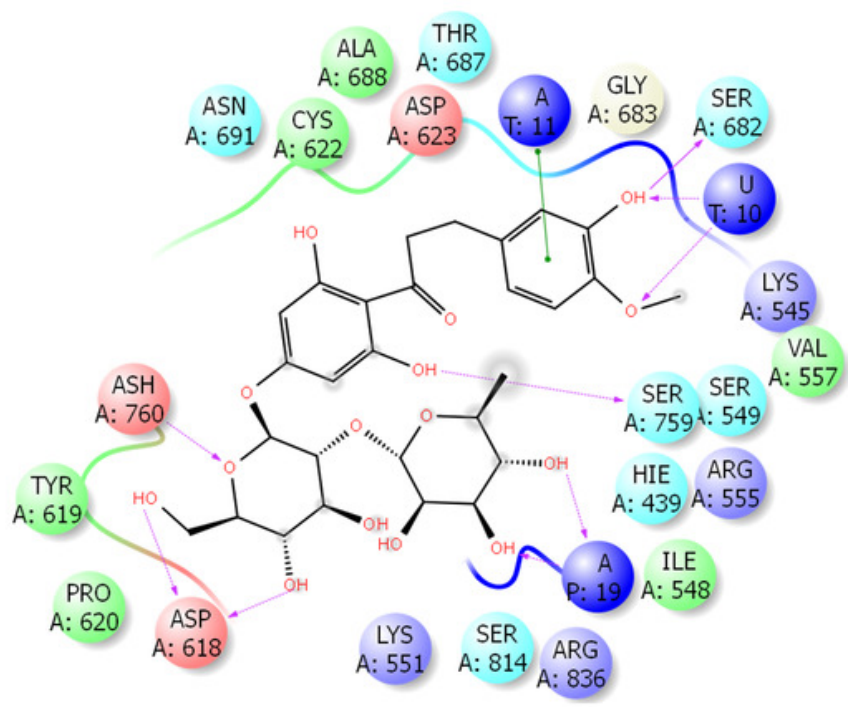




\section{Figure 7}

Molecular dynamics simulation for 100 ns.

The number of hydrogen bonds and distance from RdRP residues during 100 ns simulation of the top 2 compounds after MD simulation for 100 ns, docetaxel and neohesperidin. A) The number of hydrogen bonds formed by docetaxel and neohesperidin B) The distance between the two drugs and two selected residues in the active site during 100 ns MD simulation. The selected residues were LYS521 for docetaxel and ASP588 for neohesperidin. 


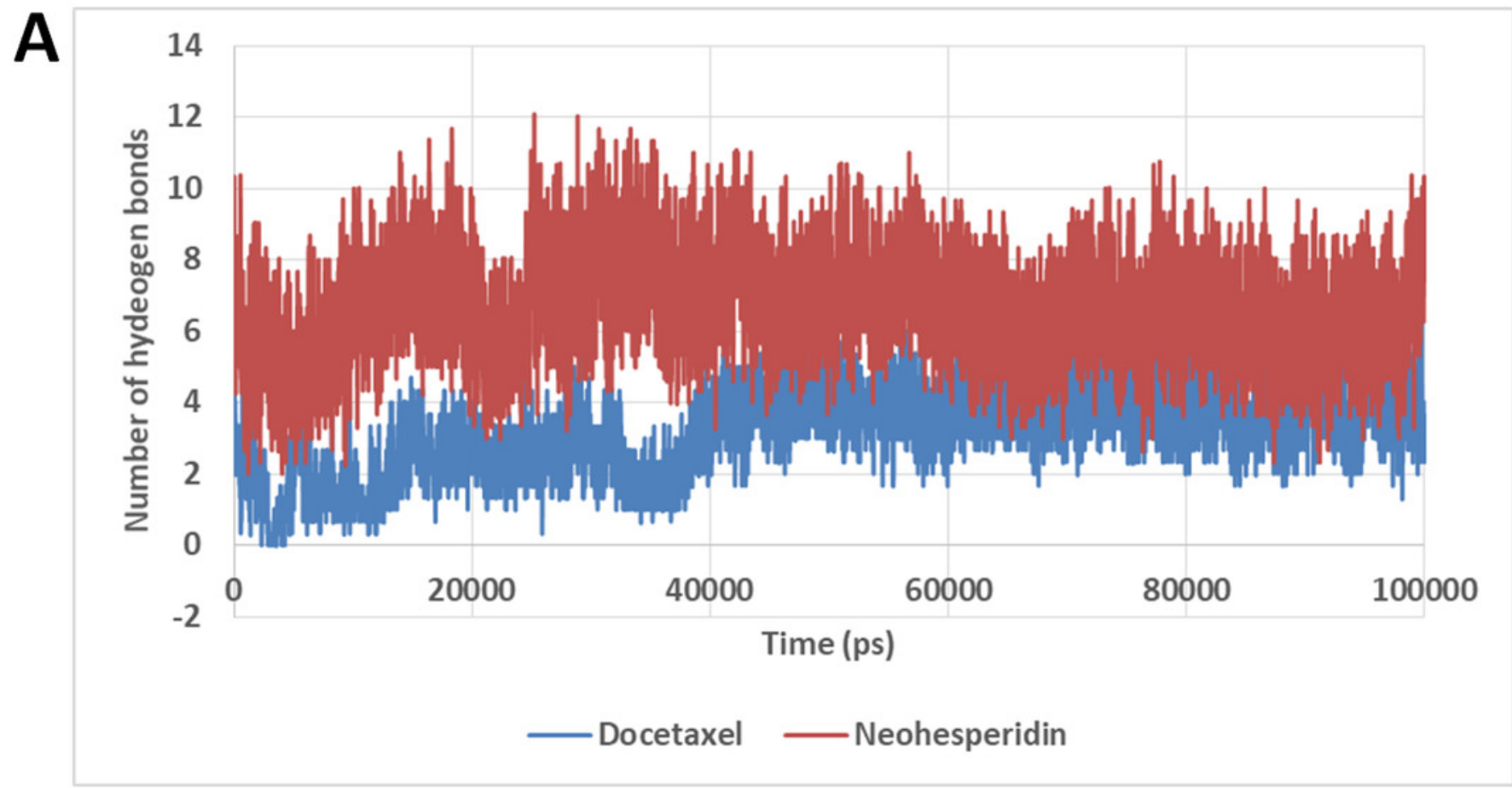

B

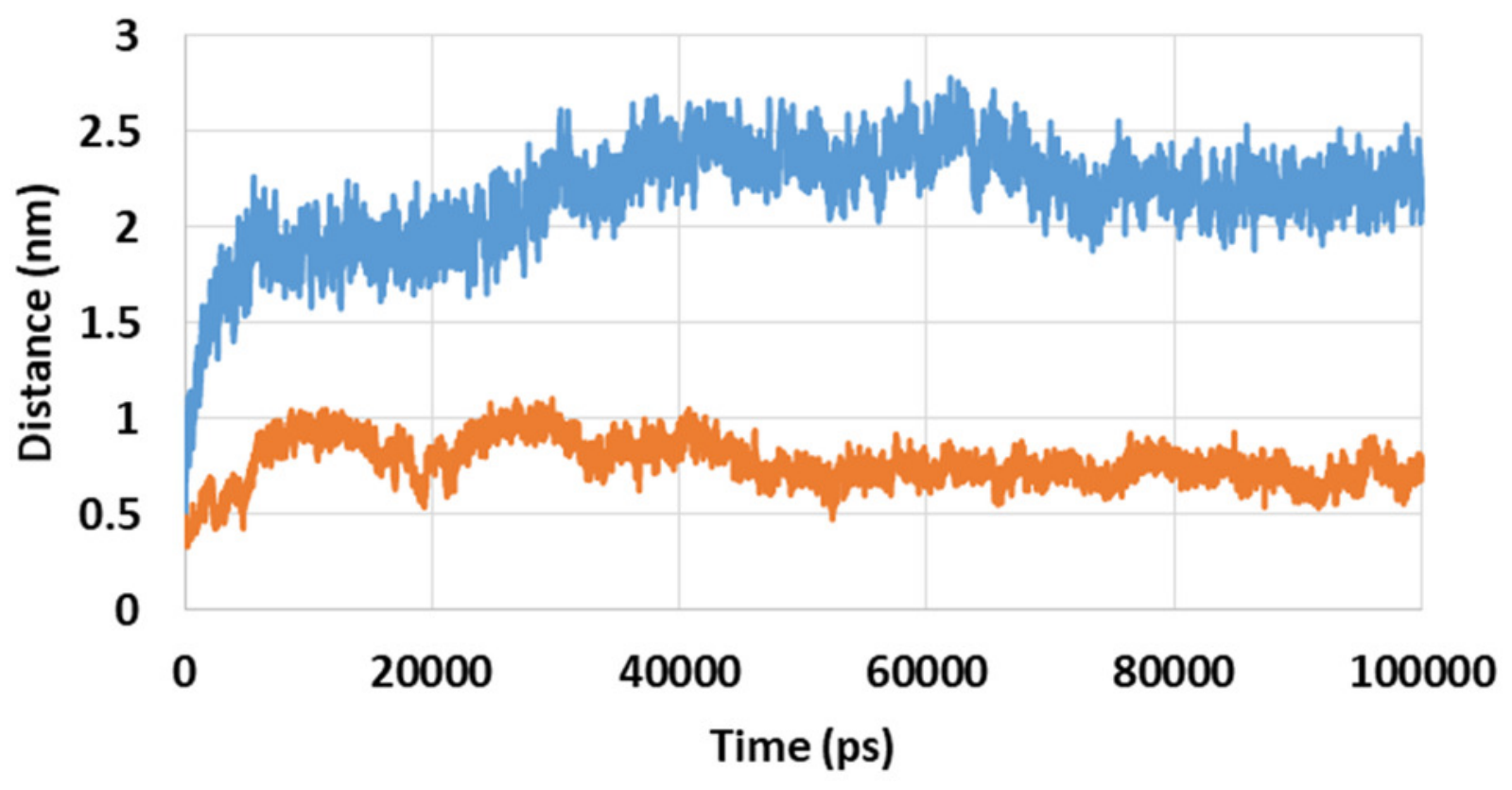

- Docetaxel - Neohesperidin 